\title{
Modified MUSIC Algorithm for DoA Estimation Using CRLH Leaky-Wave Antennas
}

\author{
Henna Paaso and Aarne Mämmelä \\ VTT Technical Research Centre of \\ Finland \\ Email: \{Henna.Paaso,Aarne.Mammela\}@vtt.fi
}

\author{
Damiano Patron and Kapil R. Dandekar \\ Department of Electrical and Computer Engineering \\ Drexel University \\ Philadelphia, PA, USA \\ Email: \{damiano.patron,dandekar\}@drexel.edu
}

\begin{abstract}
In this paper, we formulate and experimentally demonstrate direction of arrival (DoA) estimation with a two-port composite right/left handed (CRLH) leaky-wave antenna (LWA) using the multiple signal classification (MUSIC) algorithm. The CRLH LWA consists of a cascade of metamaterial unit cells, periodically modulated using varactor diodes. By changing the voltages across series and shunt varactors, the antenna is able to uniformly steer its radiation pattern. The proposed modified MUSIC algorithm, uses both antenna's input ports to estimate the DoA. Conventionally, the MUSIC algorithm needs to calculate the correlation matrix from the output vector of an array of antenna elements. In this work, we show in detail how the correlation matrix can be computed when using leaky-wave antennas. The algorithm is demonstrated using LWA in an anechoic chamber and the estimated DoA results are in good agreement with the predicted angles.
\end{abstract}

\section{INTRODUCTION}

Direction of arrival estimation using compact antenna arrays are of great importance to future cognitive radio systems. The spectrum reuse and sensing performance can be greatly improved by using spatial dimension with the help of reconfigurable antennas, DoA estimation and beamforming techniques [1], [2], [3], [4]. By using these techniques in concern in cognitive radio environments, it is possible to reduce multiple access interference and enhance the performance of cognitive users. In this paper, we formulate and experimentally demonstrate a novel modified MUSIC algorithm, which can be used to estimate the DoA of a primary user in cognitive radio networks. The size of the conventional antenna arrays is usually quite big, thus, the spatial DoA sensing may be used only in the case of the base station [5]. Given the compact and planar LWA design, the proposed DoA estimation algorithm with LWAs could be used also in small form factor devices.

DoA estimation techniques can be classified based on data analysis and implementation into four different categories: conventional methods, subspace-based methods, maximum likelihood methods, and integrated methods [6]. Conventional methods refer to beamforming and null steering techniques such as the delay-and-sum method and Capon minimum variance method. Subspace methods employ the structure of the received data, such as MUSIC [7] and estimation of signal parameters via rotational invariance technique (ESPRIT) [8]. By using MUSIC and ESPRIT algorithms, DoA resolution can

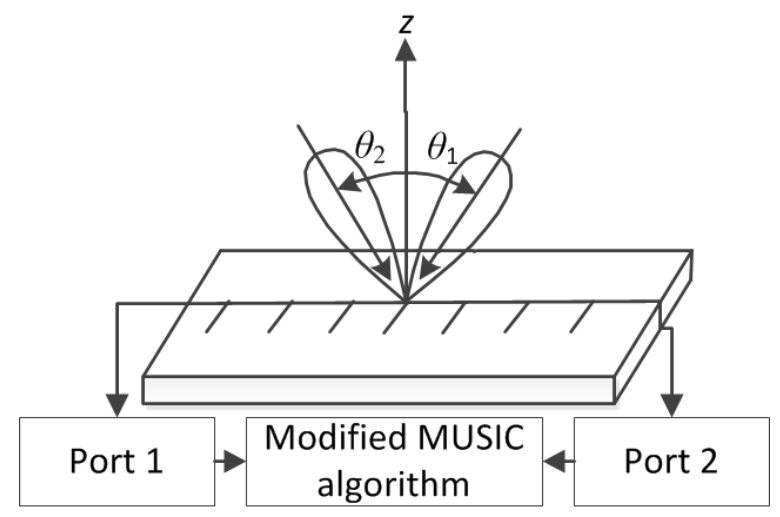

Fig. 1. System model.

be significantly improved over previous methods. In this paper we focus on the MUSIC algorithm, showing how it can be used with a metamaterial LWA instead of conventional antenna arrays.

In the literature, there are only few articles that pertain to DoA estimation with leaky-wave antennas. In [9], an energybased DoA estimation system with a LWA is presented. This system operates at a fixed frequency employing a CRLH LWA. In [10], the DoA is estimated from the cross-correlation coefficients using the antenna's received spectra with a predetermined incident angle-dependent spectra. In [11], the performance of the MUSIC DoA estimation algorithms is studied in a clustered channel model. An experimental study shows the preliminary evaluation of the algorithm using a single CRLH LWA. Unfortunately, the paper presents only experimental results and does not show how the MUSIC algorithm is formulated with LWAs. In the literature, few articles apply the electronically steerable parasitic array radiator (ESPAR) for reactance domain MUSIC algorithm [12], [13], [14]. In all these papers, the DoA estimation was performed using single port antennas.

In this paper we introduce a modified MUSIC algorithm with a two-port CRLH leaky-wave antenna, as shown in Fig. 1. The conventional MUSIC algorithm forms the correlation matrix of the groups of signal samples from antenna array elements. Since the LWA can not use the conventional MUSIC 


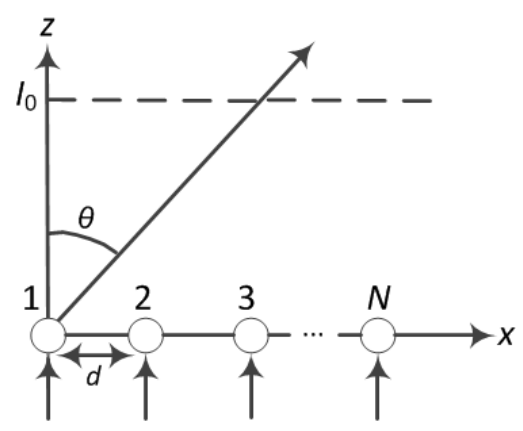

(a)

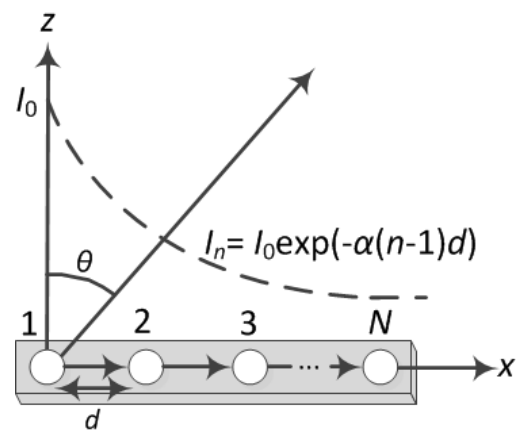

(b)

Fig. 2. Array factor. (a) Conventional uniform linear antenna array. (b) LWA.

algorithm, in this paper we show how it can be modified when using LWAs. Additionally, we show how we can make a correlation matrix using only one port, which is not presented in previous papers. In our measurements we measure the signal coming from $N$ directions, where $N$ is the number of LWA unit cells. The algorithm uses a correlation matrix that is based on these $N$ signals, collected from the two LWA's input ports.

This paper is organized as follows. Section II introduces the array factor of the LWA. Section III presents the modified MUSIC algorithm for DoA estimation using LWAs, then Section IV describes the antenna design. Measurement setup and the results are described, respectively in Section V and Section VI. Finally, conclusions are drawn in Section VII.

\section{ARRAY FACTOR OF THE LWA}

The array factor of the LWA can be formed almost in the same way as for a conventional antenna array, as shown in Fig. 2 [15]. In a conventional uniform array, the amplitude function $I_{n}$ has a constant value $I_{n}=I_{0}$. In the case of a LWA, it is an exponential function $I_{n}=I_{0} \exp (-\alpha(n-1) d)$, with leakage factor $\alpha$ and structure period $d$ [15]. Each individual LWA unit cell can be thought as a single antenna array element. Thus, the array factor can be written as [15]

$$
\begin{aligned}
A F(\theta) & =\sum_{n=1}^{N} I_{n} \exp \left(j(n-1) k_{0} d \sin (\theta)+j \xi_{n}\right) \\
& =\sum_{n=1}^{N} a_{\bmod , n}(\theta)
\end{aligned}
$$

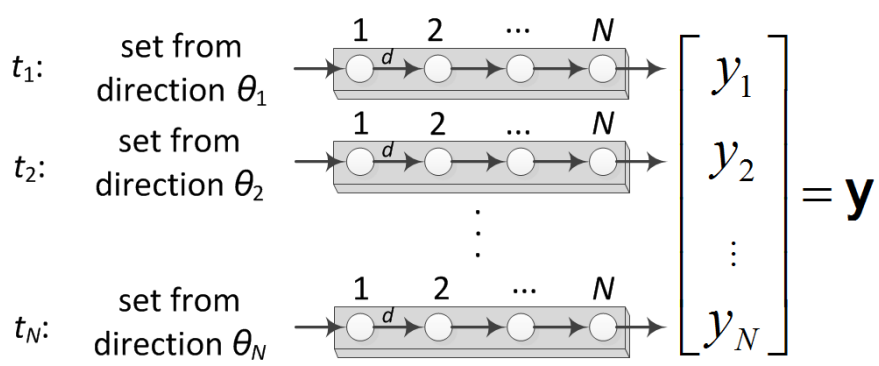

Fig. 3. LWA output computation scheme.

with $\xi_{n}=(n-1) k_{0} d \sin \left(\theta_{0}\right), N$ the number of unit cells, and $\theta_{0}$ the angular direction of the main beam. The beam angle $\theta_{0}$ is determined by $\theta_{0}=\arcsin \left(\beta / k_{0}\right)$, where $\beta$ is the propagation constant and $k_{0}$ is the free space wave number.

\section{Modified MUSIC ALGORITHM WITH LWA}

The conventional MUSIC algorithms [7] find the $D$ directions that minimize the projection of the steering vector to the noise subspace. These algorithms form a correlation matrix from the received signal coming from each antenna array element. In this paper, we show how we can make a correlation matrix using two ports of the LWA. In addition, we demonstrate how the spatial diversity of a conventional antenna array can be recreated with a LWA antenna. Specifically, we can generate spatial diversity by receiving the same signal $N$ times when the LWA switches between $N$ different radiation patterns, as shown in Fig. 3 .

Considering a LWA having $N$ unit cells, in this system we have to measure the transmitted signal $u$ from $N$ different directions. Thus, we can define each of these received signal as $y_{n}$ and a vector $\mathbf{y}$ can be represented as

$$
\mathbf{y}=\left[\begin{array}{c}
y_{1} \\
\vdots \\
y_{N}
\end{array}\right]=\mathbf{a}_{\bmod } u+\left[\begin{array}{c}
z_{1} \\
\vdots \\
z_{N}
\end{array}\right]
$$

where $z_{n}$ is an additive white gaussian noise and $\mathbf{a}_{\bmod }$ is given by

$$
\mathbf{a}_{\text {mod }}=\mathbf{A}_{\mathrm{LWA}} \mathbf{a}\left(\theta_{0}\right)
$$

where $\mathbf{A}_{\mathrm{LWA}}\left(\theta_{n}\right)$ is

$$
\mathbf{A}_{\mathrm{LWA}}=\left[\begin{array}{c}
\mathbf{a}_{\mathrm{LWA}}^{T}\left(\theta_{1}\right) \\
\mathbf{a}_{\mathrm{LWA}}^{T}\left(\theta_{2}\right) \\
\vdots \\
\mathbf{a}_{\mathrm{LWA}}^{T}\left(\theta_{N}\right)
\end{array}\right]
$$

where $T$ is transpose and

$$
\mathbf{a}_{\text {LWA }}\left(\theta_{n}\right)=\left[\begin{array}{c}
I_{1} \\
I_{2} \exp \left(j k_{0} d \sin \left(\theta_{n}\right)\right) \\
\vdots \\
I_{N} \exp \left(j(N-1) k_{0} d \sin \left(\theta_{n}\right)\right)
\end{array}\right]
$$


and

$$
\mathbf{a}\left(\theta_{0}\right)=\left[\begin{array}{c}
1 \\
\exp \left(j k_{0} d \sin \left(\theta_{0}\right)\right) \\
\vdots \\
\exp \left(j(N-1) k_{0} d \sin \left(\theta_{0}\right)\right)
\end{array}\right] .
$$

Once the received signals $y_{n}(t)$ are collected using the $N$ different radiation patterns, we can estimate the correlation matrix, which can be expressed as

$$
\hat{\mathbf{R}}_{y y}=\frac{1}{K} \sum_{k=1}^{N_{\mathrm{s}}}\left[\begin{array}{c}
y_{1}(k) \\
\vdots \\
y_{N}(k)
\end{array}\right]\left[\begin{array}{lll}
y_{1}^{*}(k) & \cdots & y_{N}^{*}(k)
\end{array}\right]
$$

where $N_{\mathrm{s}}$ is the number of received symbols for each received signal $y_{n}$, the superscript ${ }^{*}$ indicates the complex conjugate, and $K$ is the number of snapshots. Once the correlation matrix is created, we can use an eigen-decomposition to compute the noise subspace matrix $\mathbf{E}_{\mathrm{N}}$. Finally, the modified MUSIC spectrum can be generated and shown as

$$
P_{\mathrm{MUSIC}}(\theta)=\frac{1}{\mathbf{a}_{\bmod }^{H}(\theta) \mathbf{E}_{\mathrm{N}} \mathbf{E}_{\mathrm{N}}^{H} \mathbf{a}_{\bmod }(\theta)}
$$

given $-60^{\circ}<\theta<60^{\circ}$ and $H$ is Hermitian transpose. The maximum value of $P_{\text {MUSIC }}(\theta)$ will give us the estimated DoA. The flowchart in Fig. 4 summarizes the algorithm that has been described in this section.

\section{Antenna Design}

The modified MUSIC algorithm was designed to perform the DoA estimation using a reconfigurable LWA. The proposed LWA consists of 12 metamaterial CRLH unit cells, populated with varactor diodes in series and shunt configuration. By modulating the two DC bias voltages ( $\mathrm{S}-\mathrm{SH}$ ) across the varactors, the antenna is able to steer the beam around the $\mathrm{x}-\mathrm{z}$ plane. The single unit cell has been optimized in left hand regime in order to provide the maximum space coverage when using each port. In other words, by exciting Port 1 the beam can be steered from $0^{\circ}$ to $+60^{\circ}$, while by switching to Port 2 , it covers the symmetric angles from $0^{\circ}$ to $-60^{\circ}$. In Table I we summarize the DC configurations with relative beam angle and antenna gain. We considered $10^{\circ}$ to be the spatial resolution between each beam.

The design was etched on a low loss Rogers 4360 substrate having dielectric constant $\epsilon_{r}=6.15$. In terms of DC biasing, the $\mathrm{S}$ voltage is provided to the series varactors through folded and lumped $(220 \mathrm{nH})$ inductors while the $\mathrm{SH}$ voltage to the shunt varactors are provided using folded open stubs. In order to decouple the two voltages, a $0.5 \mathrm{pF}$ capacitor has been added in series to the shunt varactor. The high dielectric constant and design of small RF chokes, allowed for a compact layout. The overall dimensions are $L=15.6 \mathrm{~cm}, H=3.8 \mathrm{~cm}$, as shown in Fig. 5.

Through a full wave electromagnetic simulator (HFSS), the design has been tuned to operate from $2.41 \mathrm{GHz}$ to 2.48 $\mathrm{GHz}$, covering the entire $2.4 \mathrm{GHz} \mathrm{WiFi}$ band. The impedance integrity between the feed line and the antenna's input ports

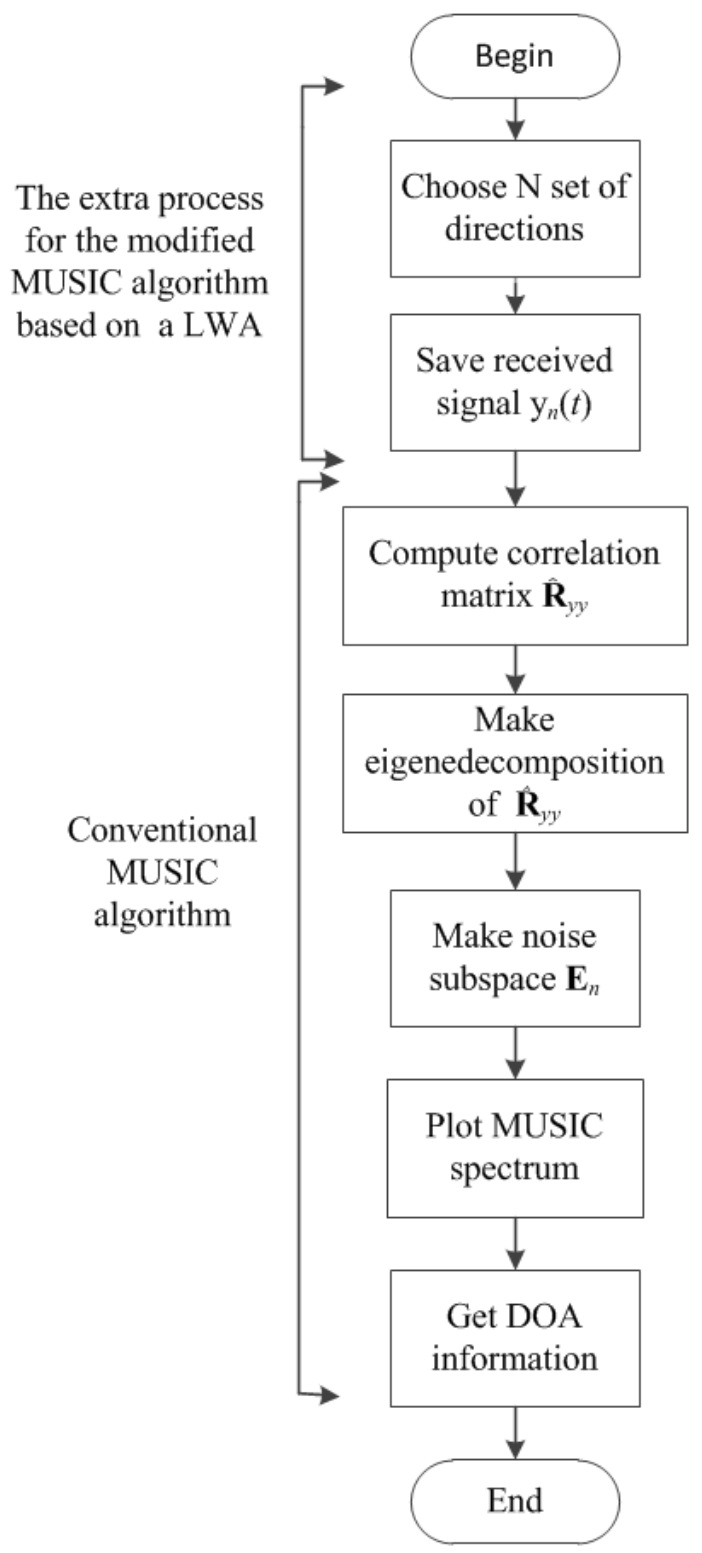

Fig. 4. Flowchart of the modified MUSIC algorithm with LWAs.

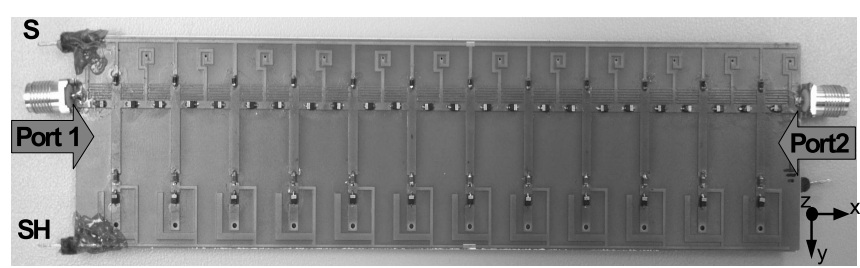

Fig. 5. CRLH LWA with RF input ports and S-SH DC bias. $L=15.6 \mathrm{~cm}$, $H=3.8 \mathrm{~cm}$.

was measured using an Agilent N5230A. The return loss $\left(\mathrm{S}_{11^{-}}\right.$ $\mathrm{S}_{22}$ ) exhibit values below $-10 \mathrm{~dB}$ over the entire bandwidth of operation while the insertion loss between the two ports $\left(\mathrm{S}_{12}\right)$, 
TABLE I

LWA CONFIGURATIONS

\begin{tabular}{|c|c|c|}
\hline Beam Angle & Bias Voltages & Gain \\
\hline $0^{\circ}$ & $\mathrm{S}=12 \mathrm{~V}, \mathrm{SH}=13.7 \mathrm{~V}$ & $4.1 \mathrm{~dB}$ \\
\hline $\pm 10^{\circ}$ & $\mathrm{S}=13.7 \mathrm{~V}, \mathrm{SH}=13.7 \mathrm{~V}$ & $3.9 \mathrm{~dB}$ \\
\hline $\pm 20^{\circ}$ & $\mathrm{S}=14.4 \mathrm{~V}, \mathrm{SH}=15.8 \mathrm{~V}$ & $4.7 \mathrm{~dB}$ \\
\hline $\pm 30^{\circ}$ & $\mathrm{S}=16.5 \mathrm{~V}, \mathrm{SH}=16.5 \mathrm{~V}$ & $4.5 \mathrm{~dB}$ \\
\hline $\pm 40^{\circ}$ & $\mathrm{S}=20.2 \mathrm{~V}, \mathrm{SH}=18.3 \mathrm{~V}$ & $3.7 \mathrm{~dB}$ \\
\hline $\pm 50^{\circ}$ & $\mathrm{S}=27.8 \mathrm{~V}, \mathrm{SH}=18.3 \mathrm{~V}$ & $4.2 \mathrm{~dB}$ \\
\hline $\pm 60^{\circ}$ & $\mathrm{S}=29.5 \mathrm{~V}, \mathrm{SH}=20 \mathrm{~V}$ & $2 \mathrm{~dB}$ \\
\hline \multicolumn{3}{|c}{} \\
\hline
\end{tabular}

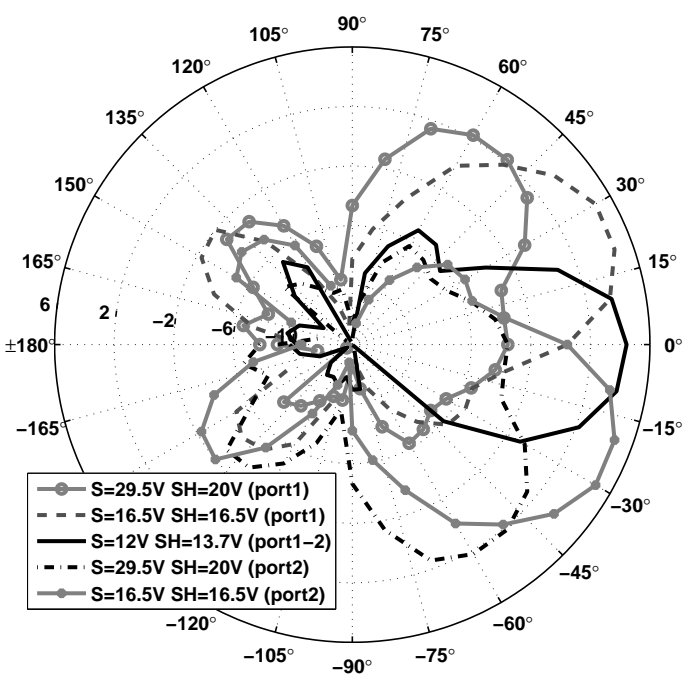

Fig. 6. LWA measured radiation patterns (x-z plane) at frequency $2.46 \mathrm{GHz}$.

is always between $10 \mathrm{~dB}$ and $15 \mathrm{~dB}$.

Using our anechoic chamber facility, radiation patterns of all configurations were collected from $2.452 \mathrm{GHz}$ to $2.472 \mathrm{GHz}$. In Fig. 6, we show five beams. The positive beam angles were taken by connecting Port 1 and negative beam angles were taken by connecting Port 2 . The measured gain is relatively constant $(4 \mathrm{~dB}$ to $5 \mathrm{~dB}$ ) for all the configurations within the range of $0^{\circ}$ and $\pm 50^{\circ}$. However, a small gain reduction was experienced for angles above $\pm 60^{\circ}$, as a consequence of the fact that the propagation constant along the structure starts to approach the guided regime.

\section{Measurement gonfiguration}

In order to evaluate the performance of the proposed algorithm, the experiment was carried out in an anechoic chamber using the set up shown in Fig. 7. We used the CRLH LWA to transmit a 64 subcarriers signal within the frequency range from $2.452 \mathrm{GHz}$ to $2.472 \mathrm{GHz}$. The LWA's length of the one cell is $d=1.3 \mathrm{~cm}$ and the leakage factor $\alpha=1$. At the receiver, a horn antenna was connected in series to a lownoise amplifier. The signal was transmitted from 12 different directions $\left(-60^{\circ}\right.$ to $+50^{\circ}$ in steps of $\left.10^{\circ}\right)$ by setting the bias voltages $\mathrm{S}$ and $\mathrm{SH}$. We considered four different DoA angles during measurements: $15^{\circ}, 25^{\circ}, 35^{\circ}$ and $45^{\circ}$. The different DoAs were taken by tilting the CRLH LWA by an angle $\theta_{0}$, as shown in Fig. 7.

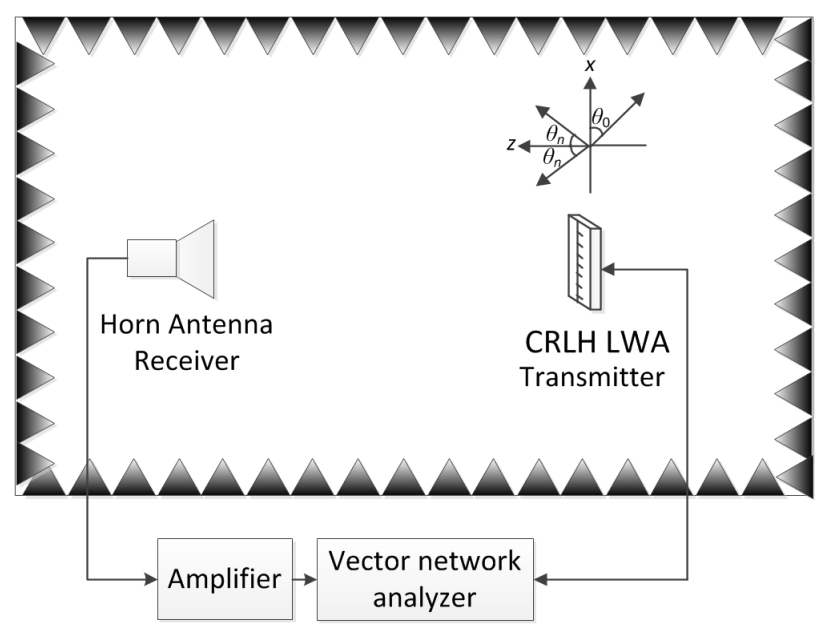

Fig. 7. Anechoic chamber measurements setup.

We first connected the feed port to the LWA's Port 1 and collected channel measurements for all the seven LWA configurations and for each DoA. After that, we switched to the second antenna port and replicated the same set of measurements. We assumed the wireless channel to be reciprocal because of the anechoic chamber environment. The modified MUSIC spectrum is calculated by averaging over the number of subcarriers as shown in (9)

$$
P_{\mathrm{avg}}(\theta)=\frac{1}{N_{\mathrm{Sc}}} \sum_{n=1}^{N_{\mathrm{Sc}}} \frac{1}{\mathbf{a}_{\mathrm{mod}}^{H}(\theta) \mathbf{E}_{\mathrm{N}} \mathbf{E}_{\mathrm{N}}^{H} \mathbf{a}_{\text {mod }}(\theta)} .
$$

\section{EXPERIMENTAL RESULTS}

The goal of our practical experiment was to estimate the DoA using the proposed algorithm. The plots from Fig. 8 to Fig. 11 show the power spectrum as function of the DoA angles. In each plot, the two curves are related to two different scenarios based on the switching between the LWA's ports. In the first one (solid curve), we collected signals using the following scheme: Port 2 receives signals from directions: $-60^{\circ},-50^{\circ},-40^{\circ},-30^{\circ},-20^{\circ},-10^{\circ}$ and Port 1 receives signals from directions: $0^{\circ}, 10^{\circ}, 20^{\circ}, 30^{\circ}, 40^{\circ}, 50^{\circ}$. In the second scenario (dashed curve): Port 2 receives signals from directions: $-60^{\circ},-50^{\circ},-40^{\circ},-30^{\circ},-20^{\circ},-10^{\circ}, 0^{\circ}$ and Port 1 receives signals from directions: $10^{\circ}, 20^{\circ}, 30^{\circ}, 40^{\circ}$, $50^{\circ}$.

From the results summarized in Table II, we see that the proposed algorithm based on the LWA provides DoA values very close to the expected angles. However, the solid trace relative to Scenario 1 experiences slightly high error for angles above $25^{\circ}$. The source of this error may be due to the manufacturing process of the LWA. Specifically, during the milling process, it is difficult to avoid some discrepancies in the width of the very small interdigital capacitors that are part of the LWA's unit cell. Therefore, between the two opposite sides of the structure, the propagation constant may also be slightly different, causing the beams to not be perfectly 
TABLE II

DOA MEASUREMENT RESULTS

\begin{tabular}{|c|c|c|c|c|}
\hline Expected DoA & \multicolumn{2}{|c|}{ Scenario 1 (solid) } & \multicolumn{2}{c|}{ Scenario 2 (dashed) } \\
\hline & DoA & Error & DoA & Error \\
\hline $15^{\circ}$ & $16^{\circ}$ & $0.8 \%$ & $17^{\circ}$ & $1.6 \%$ \\
\hline $25^{\circ}$ & $20^{\circ}$ & $4.1 \%$ & $25^{\circ}$ & $0.0 \%$ \\
\hline $35^{\circ}$ & $26^{\circ}$ & $5.8 \%$ & $35^{\circ}$ & $0.0 \%$ \\
\hline $45^{\circ}$ & $38^{\circ}$ & $18.4 \%$ & $47^{\circ}$ & $1.7 \%$ \\
\hline Average & & $\mathbf{1 8 . 1} \%$ & & $\mathbf{3 . 3 \%}$ \\
\hline
\end{tabular}

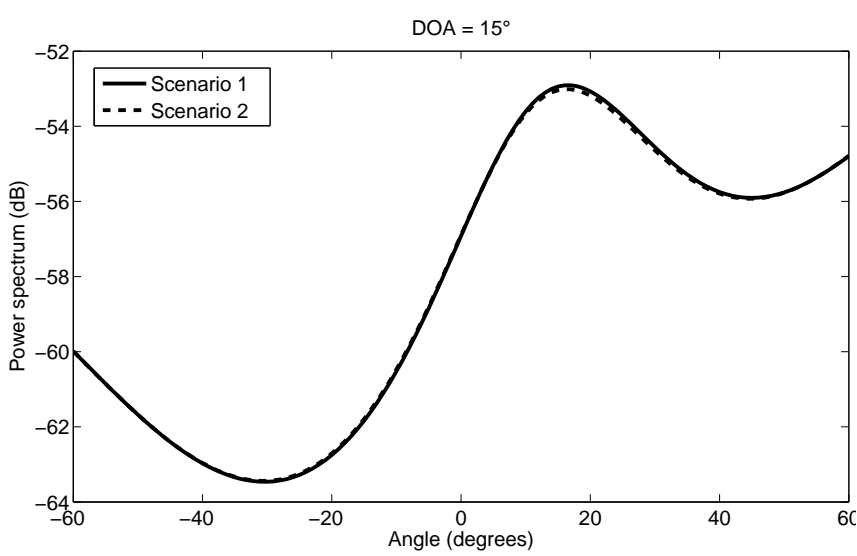

Fig. 8. Modified MUSIC spectrum for the case where DoA is $15^{\circ}$.

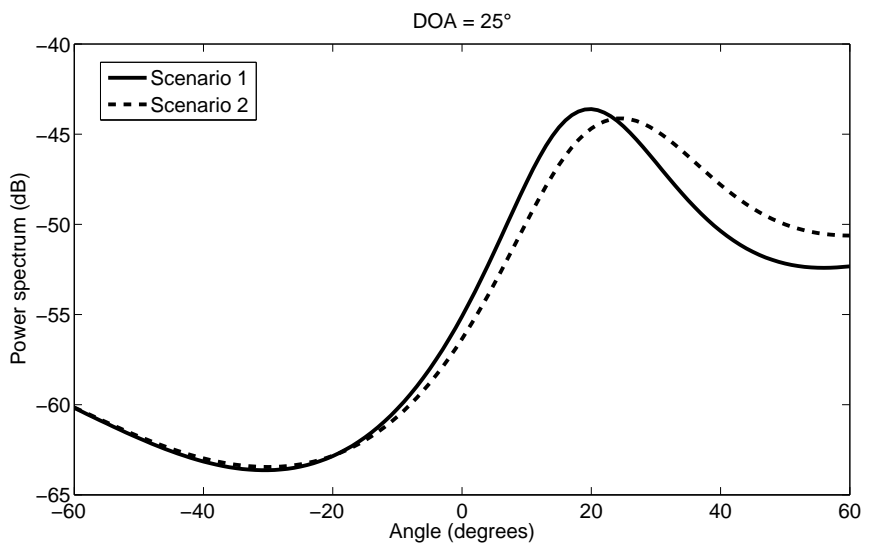

Fig. 9. Modified MUSIC spectrum for the case where DoA is $25^{\circ}$.

symmetrical when switching between the two input ports. Besides this small discrepancy, we can see that the dashed trace for Scenario 2 is in very good agreement with the expected DoAs, demonstrating the value of our approach. Due to the fact that the LWA creates spatial diversity in a different way compared to conventional antenna arrays, the proposed algorithm results in a wider peak of the power spectrum with respect to conventional methods. Moreover, this algorithm takes advantage of a single antenna element capable of beamsteering with LWAs instead of using antenna arrays. Therefore, due to the absence of complex feeding networks and multiple radiating elements, this approach results to be more cost-effective and space saving.

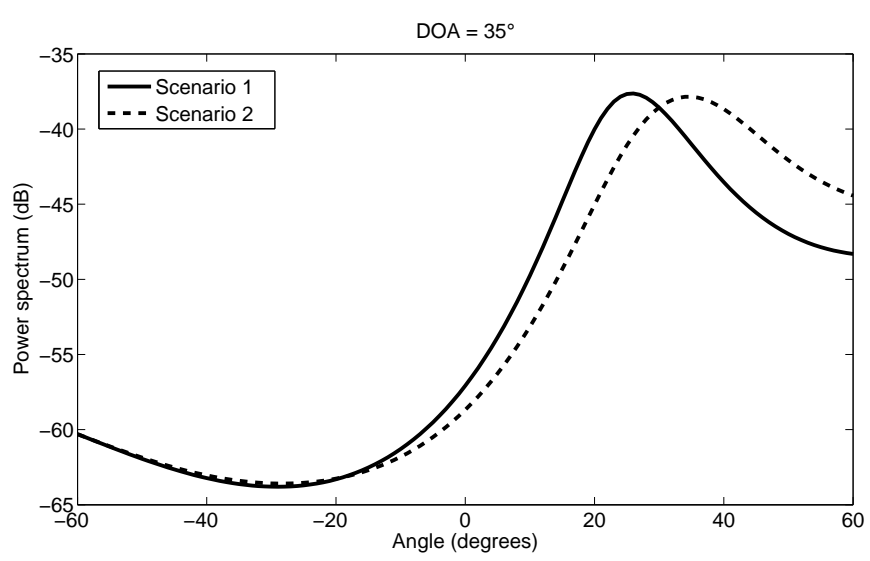

Fig. 10. Modified MUSIC spectrum for the case where DoA is $35^{\circ}$.

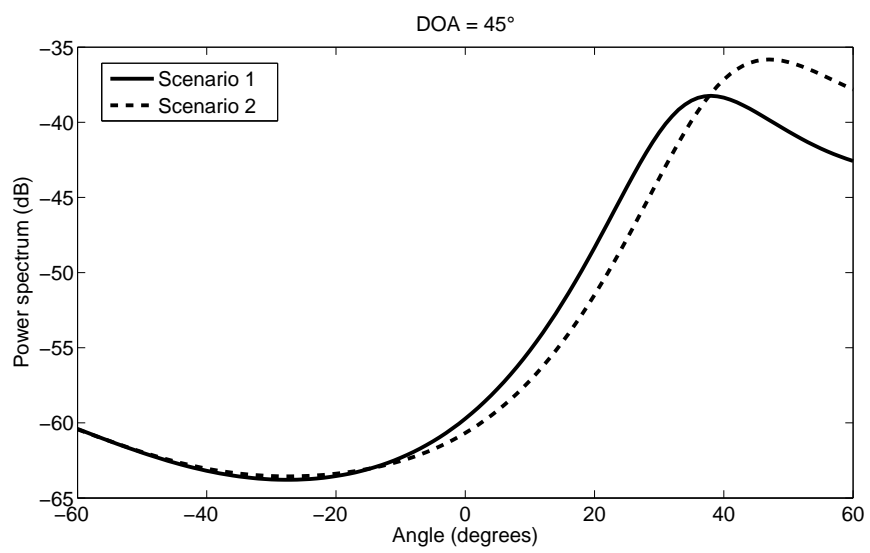

Fig. 11. Modified MUSIC spectrum for the case where DoA is $45^{\circ}$.

\section{CONCLUSION}

In this work, we have proposed a novel modified MUSIC algorithm for DoA estimation based on a two-port CRLH reconfigurable leaky-wave antenna. The LWA is able to steer a directional beam by modulating the propagation constant through the DC biasing of varactor diodes. By taking advantage of the LWA's compact dimension and pattern reconfigurability, we developed a modified MUSIC algorithm for direction of arrival estimation. We have shown how the correlation matrix can be computed when using LWAs and its integration within the MUSIC algorithm. Finally, an experimental performance evaluation has been carried out by using a CRLH LWA within an anechoic chamber facility. The algorithm results show good agreement with the predicted DoA angles, demonstrating that the DoA estimation of the impinging wave can be successfully performed by using the two ports of a CRLH LWA. The proposed algorithm for DoA estimation along with the planar and compact LWA layout, can be valuable solution to enhance the performance of cognitive radio systems. Additionally, LWAs have many other advantages over conventional antenna arrays such as a low cost, low-power consumption due to the absense of complex feeding network. Our future work will involve more antenna 
designs and the realization of a new LWA having narrower half power beamwidth (HPBW) and enhanced beam symmetry to improve the estimated DoA accuracy.

\section{ACKNOWLEDGMENT}

This work has been performed in the framework of the REgonfigurable Antenna based enhancement of Dynamic Spectrum access algorithms (READS) project, which is funded VTT Technical Research Centre of Finland and the Finnish Funding agency for Technology and Innovation (Tekes). We would like to thank Adant S.r.l. for the antenna prototypes. The work has been carried out in Drexel University. This work was also supported by CNS-1147838 from the U.S. National Science Foundation as part of the Wireless Innovation between Finland and United States (WiFiUS) partnership.

\section{REFERENCES}

[1] S. Wang, Q. Liu, and Z. Zhou, "Cooperative cognitive radio network using smart antenna," in Proc. IEEE International Conference Communications Technology and Applications, 2009, pp. 450-456.

[2] J. Xie, Z. Fu and H. Xian, "Spectrum sensing based on estimation of direction of arrival," in Proc. International Conference Computational Problem-Solving, 2010, pp. 39-42.

[3] E. W. T. Lee and A. P. Vinod, "A spatial spectral detection approach for primary user interference mitigation in cognitive radios," in Proc. XXXth URSI, General Assembly and Scientific Symposium, 2011, pp. 1-4.

[4] R. J. Weber, "Development of a cognitive array system," Masters Thesis, Montana State University, 2010.

[5] P. Gardner, M. R. Hall, J. Kelly, F. Ghaneml, and E. Ebrahim, "Reconfigurable antennas for cognitive radio: requirements and potential design approaches," in Proc. Institution of Engineering and Technology Seminar on Wideband, Multiband Antennas and Arrays for Defence or Civil Applications, 2008, pp. 89-94.

[6] C. A. Balanis, Antenna Theory - Analysis and Design. 3rd ed. U.S.A: John Wiley \& Sons, 2005

[7] R. O. Schmidt, "Multiple emitter location and signal parameter estimation," in Proc. RADC Spectrum Estimation Workshop, 1979 pp. $243-258$.

[8] R. Roy, "ESPRIT: Estimation of signal parameters via rotational invariance techniques," Ph.D. Dissertation, Stanford University, Stanford, California, 1987.

[9] S. Abielmona, H. V. Nquen, and C. Caloz, "Analog direction of arrival estimation using an electronically-scanned CRLH leaky-wave antenna," IEEE Transactions on Antennas and Propagation, vol. 59, no. 4, pp. 1408-1410, April 2011.

[10] X. Yu and H. Xin, "Direction of arrival estimation utilizing incident angle depends spectra," Proc. IEEE International Microwave Symposium Digest (MTT), 2012, pp. 1-3.

[11] V. Vakilian, J. F. Frigon, and S. Roy, "Direction-of-arrival estimation in a clustered channel model," in Proc. IEEE 10th International New Circuits and Systems Conference, 2012, pp. 313-316.

[12] C. Plapous, J. Cheng, E. Taillefer, H. Akifumi and T. Ohira, "Reactancedomain MUSIC for ESPAR Antennas," in Proc. IEEE 33rd European Microwave Conference, 2003, pp. 793-796.

[13] C. Sun and N. C. Karmakar, "Direction of arrival estimation based on a single port smart antenna using MUSIC algorithm with periodic signals," International Journal of Signal Processing, vol. 1, number 2, pp. 153-162, 2004.

[14] E. Taillefer, C. Plapois, C. Cheng, K. Igusa, and T. Ohira, "Reactancedomain MUSIC for ESPAR Antennas (experiment)," in Proc. IEEE Wireless Communications and Networking, 2003, pp. 98-102.

[15] C. Caloz and T. Itoh, Electromagnetic Metamaterials Transmission Line Theory and Microwave Applications, NJ, U.S.A: John Wiley \& Sons, 2006. 